phys. stat. sol. (a) 153, K9 (1996)

Subject classification: $75.50 ; 75.60 ; \mathrm{S} 10$

Institute of Physics, National Taiwan Normal University, Taipei ${ }^{1}$ ) (a),

Institute of Physics, Academia Sinica, Taipei ${ }^{2}$ ) and

Department of Physics, National Chung Cheng University, Chiayi $\left.{ }^{3}\right)(b)$, and

Institute of Physics, National Taiwan University, Taipei ${ }^{4}$ ) (c)

\title{
Low Temperature Magnetization of a Ferrofluid of Magnetite Coated with Polysterene
}

By

I. J. JANG (a), Y. D. YAO (b), I. KliK (b), H. E. Horng (a), and H. C. YANG (c)

(Received May 9, 1995; in revised form October 12, 1995)

Physical phenomena associated with magnetic fluids have, over the years, attracted considerable attention; we wish to report here on unusual behavior observed in thermomagnetic curves of a suspension of polysterene coated magnetic particles in distilled water. The ferrofluid was produced by Polysciences Inc., USA, the weight fraction of the magnetic particles is approximately $2.5 \%$ and the ratio of magnetite to polystyrene is approximately $1: 1$ by weight. The ferrofluid composition was analyzed by an energy dispersive $\mathrm{X}$-ray spectrometer (EDS) and only $\mathrm{Fe}, \mathrm{O}$, and $\mathrm{C}$ were found. Using SEM (JEOL Model 6300) we determined that the particles are spherical (diameter 100 to $1200 \AA$, mean 600 to $700 \AA$ ) so that their magnetic shape anisotropy may be unimportant. The sample magnetization below $300 \mathrm{~K}$ was measured by a vibrating sample magnetometer (VSM) with resolution of $10^{-7} \mathrm{~A} \mathrm{~m}^{2}$. Using a closed cycle refrigerator we carried out thermomagnetic measurements between $T=35 \mathrm{~K}$ and room temperature both under zero field cooling (ZFC) and under field cooling (FC) regimes. The rate of temperature change was approximately $2 \mathrm{~K} / \mathrm{min}$.

At $300 \mathrm{~K}$ the ferrofluid has zero remanence $\left(M_{\mathrm{r}}\right)$ and coercivity $\left(H_{\mathrm{c}}\right)$, with magnetization reversals taking place by both Brownian rotation of the particles [1] and by Néel's rotation of the magnetization vector [2]. At $35 \mathrm{~K}$, on the other hand, the carrier liquid is frozen, Brownian rotation is blocked and Néel's rotation is slowed down. The sample magnetization thus cannot instantaneously follow the applied field and hysteresis follows, with $M_{\mathrm{r}}=0.26 \mathrm{~A} \mathrm{~m}^{2} / \mathrm{kg}$ and $H_{\mathrm{c}}=170 \mathrm{Oe}$ (see Fig. 1). In order to avoid unwieldy numerical expressions we use here Oersted and note that $4 \pi \mathrm{Oe}=10^{3} \mathrm{~A} / \mathrm{m}$ in SI units.

The observed thermomagnetic curves of ZFC and FC runs are shown in Fig. 1 for applied fields between 50 and 1000 Oe, with the sample cooled from its initial state at room temperature. Below $273 \mathrm{~K}$ the measured curves resemble the behavior of noninteracting small particles [3] whose magnetization reverses via thermal (Néel) activation [2]. In particular, the

\footnotetext{
1) Taipei 117, Taiwan/R.O.C.

2) Taipei 115, Taiwan/R.O.C.

${ }^{3}$ ) Chiayi 621, Taiwan/R.O.C

${ }^{4)}$ Taipei 107, Taiwan/R.O.C.
} 


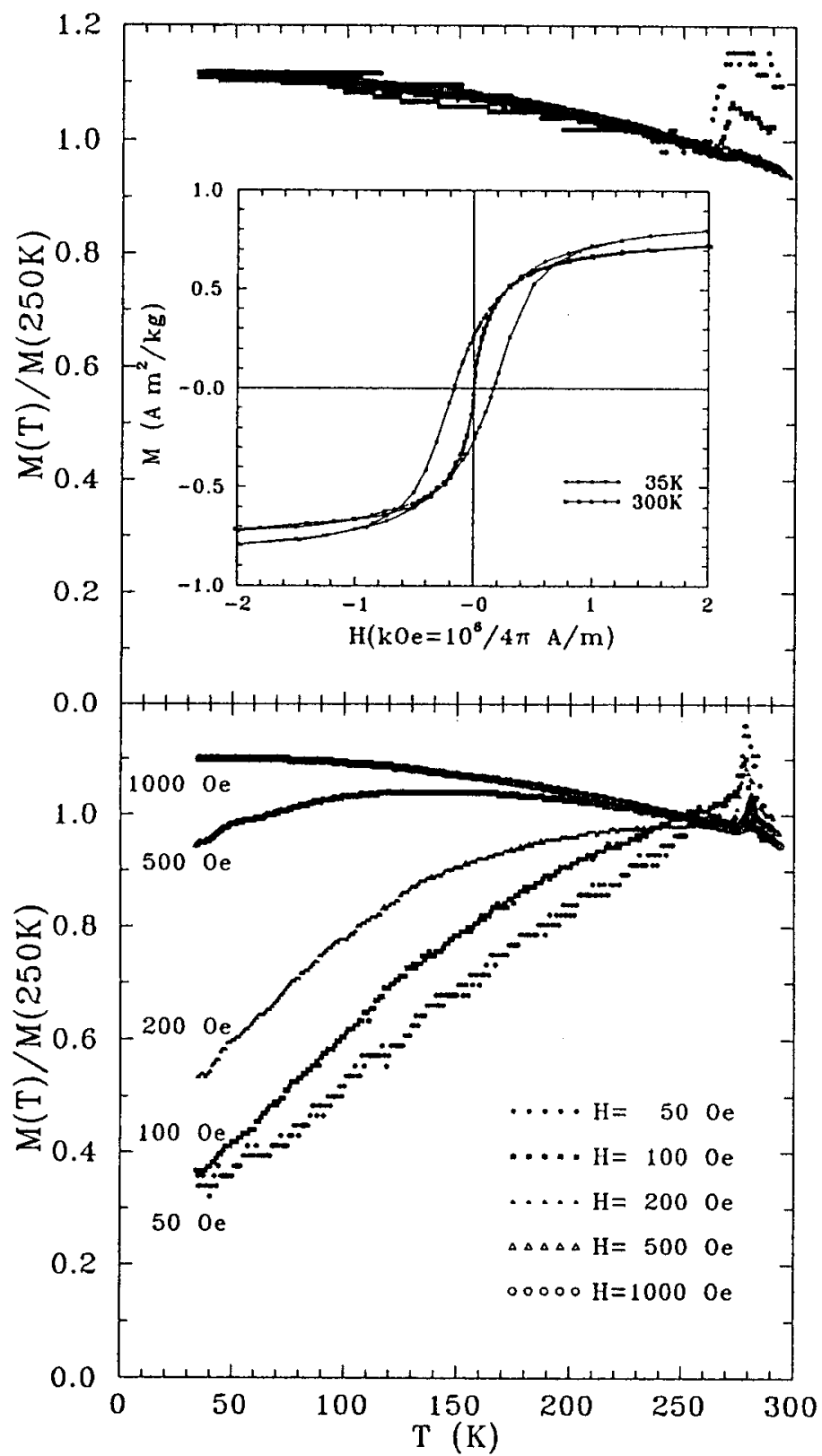

Fig. 1. Scaled thermomagnetic curves for FC (above) and ZFC (below) samples at the indicated applied field. Inset shows hysteresis curves of the ferrofluid at $300 \mathrm{~K}$ (zero coercivity) and $35 \mathrm{~K}$

ZFC curves display the typical decrease in the blocking temperature $T_{\mathrm{b}}$ with increasing applied field and $T_{\mathrm{b}}<35 \mathrm{~K}$ is here outside the observed temperature range for an applied field of $1000 \mathrm{Oe}$. An ensemble of identical particles would exhibit a sharp local maximum around $T_{\mathrm{b}}$ while a distribution of particle sizes leads to the observed broad peaks [4]. 


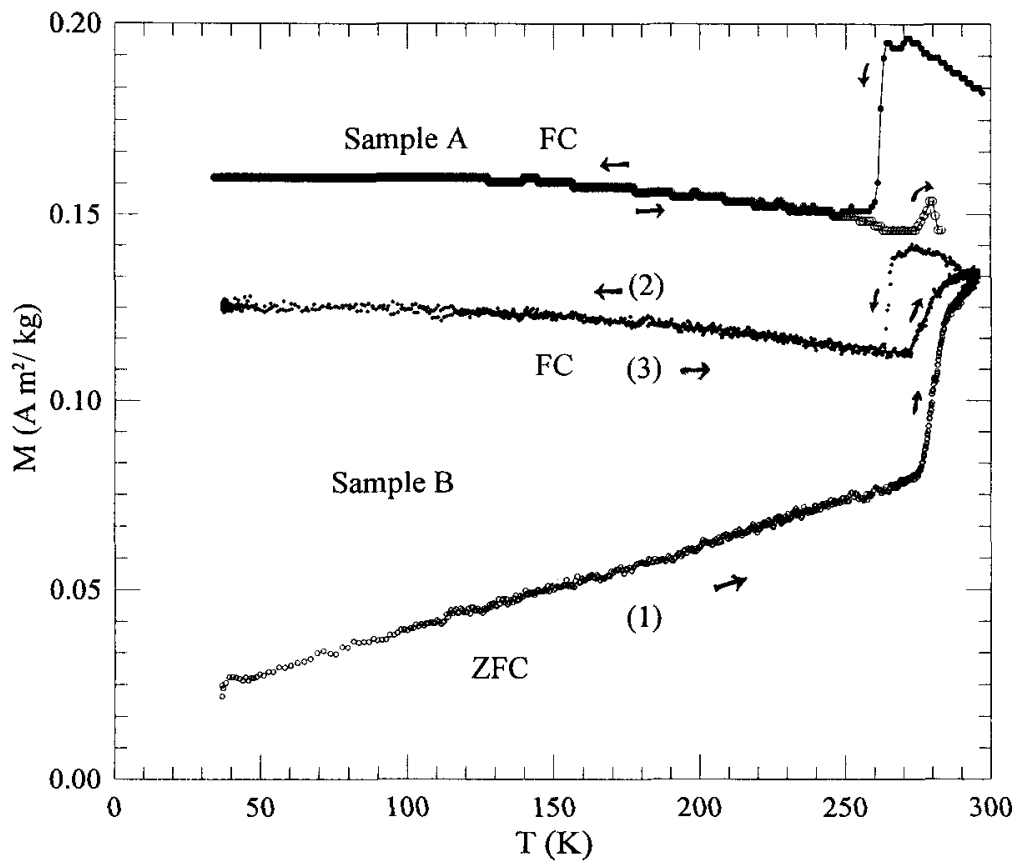

Fig. 2. A detailed plot of thermomagnetic curves in applied field of 30 Oe. Shown is, for sample A, an FC run from 300 to $35 \mathrm{~K}$ and vice versa as indicated by arrows and, for sample $\mathrm{B}$, one ZFC run and two subsequent FC runs with decreasing, and then again increasing temperatures (arrows and numbers). The two samples differ by volume

The data presented so far are standard. However, in our measurements we also encountered a prominent peak located in the field vicinity of the freezing point of the carrier fluid, independent of the applied field. Moreover, these peaks exhibit also striking hysteresis behavior illustrated by the detailed plots of Fig. 2. We explain the presence of these peaks and their hysteresis properties as being due to alignment of particle chains in the carrier fluid. These chains arise due to mutual dipole-dipole interactions between the particles and in the carrier liquid they are approximately aligned with applied field while in zero field their orientation is random. The significant magnetization enhancement observed in the ZFC run for sample B (Fig. 2) is easily explained by alignment of the initially randomly oriented particles and chains once they are free to rotate in the carrier liquid. In the subsequent FC run (2) the sample magnetization precipitously decreases as the carrier fluid becomes frozen again and we assume that ice crystal formation leads to misalignment and tearing apart of the particle chains which are then restored during the final FC run (3). In other words, the freezing process impairs the particle alignment and changes the detailed magnetic structure of the sample, a conclusion which is supported also by the two FC runs on sample A (Fig. 2).

The present simple model is sufficient to explain the overall observed hysteresis behavior of the ferrofluid as it passes between its frozen and liquid states. However, the detailed structure of the observed hysteresis effect cannot be explained within this sim- 
ple model only and we conjecture that long-range critical fluctuations accompanying the first-order phase transition in the carrier fluid contribute to the chain alignment. A detailed study is required to confirm this point.

\section{References}

[1] J. Frenkel, The Kinetic Theory of Liquids, Dover, New York 1955.

[2] L. NÉEL, C. R. Acad. Sci. (France) 228, 664 (1949).

[3] I. Klik, C. R. Chang, and H. L. Huang, Phys. Rev. B 47, 8605 (1993).

[4] J. S. YAng, J. LeE, I. KliK, and C. R. Chang, J. Magnetism magnetic Mater. 140/144, 407 (1995). 